\author{
Jingru Song \\ State-Key Laboratory of Nonlinear Mechanics, \\ Institute of Mechanics, \\ Chinese Academy of Sciences, \\ Beijing 100190, China \\ e-mail: songjingru@Inm.imech.ac.cn

Yueguang Wei
Department of Mechanics and
Engineering Science,
College of Engineering,
Peking University,
Beijing 100871, China
e-mail: weiyg@pku.edu.cn

\section{A Method to Determine Material Length Scale Parameters in Elastic Strain Gradient Theory}

With specimen size decrease for advanced structural materials, the measured mechanics behaviors display the strong size effects. In order to characterize the size effects, several higher-order theories have been presented in the past several decades, such as the strain gradient theories and the micro-polar theories, etc. However, in each higher-order theory, there are several length scale parameters included, which are usually taken as the material parameters and are determined by using the corresponding theoretical predictions to fit experimental results. Since such kind of experimental approaches needs high techniques, it is very difficult to be performed; therefore, the obtained experimental results are very few until now; in addition, the physical meanings of the parameters still need to be investigated. In the present research, an equivalent linkage method is used to simply determine the elastic length parameters appeared in the elastic strain gradient theory for a series of typical metal materials. We use both the elastic strain gradient theory and the higher-order Cauchy-Born rule to model the materials mechanics behaviors by means of a spherical expanding model and then make a linkage for both kinds of results according to the equivalence of strain energy densities. The values of the materials length parameters are obtained for a series of typical metal systems, such as the face-centered cubic (FCC), body-centered cubic (BCC), and hexagonal close-packed (HCP) metals. [DOI: $10.1115 / 1.4045523$ ]

Keywords: mechanical behavior, material length scale, strain gradient theory, CauchyBorn rule, constitutive modeling of materials

\section{Introduction}

In the past several decades, with the development of researches on the mechanical behavior of advanced structural materials (ASMs), more and more new phenomena about ASMs, such as micro-/nanostructured materials, etc., have been displayed. One of the marked phenomena is that the smaller the specimen size, the stronger the materials strength. These features are called the size effect. Experiments at the micron/sub-micron scale have revealed that the size-dependent strengthening is associated with the strain gradient (SG) effects induced by non-uniform deformation at micro- or nanoscale, for example, bending of thin beams whether or elastic plastic [1-4], torsion of thin wires [5,6], and indentation of materials [7-9]. However, the conventional mechanics theory (CMT) cannot predict these size effects effectively because no length scales are reflected in the constitutive model of the CMT. In order to describe the size effects, different versions of SG theories have been presented [10-18]. In these SG theories, the strain gradient contributions appearing in the constitutive equations match with the conventional strain terms by introducing a kind of length parameters which are called material length scale parameters $\ell$. The measurability of the material length scale parameters in SG theories should be very important. However, since such kind of experimental approach commonly needs high techniques, it is very difficult to carry out the experiments of measuring the material length scale parameters; therefore, the obtained experimental results are very few, in addition, the physical meanings of the parameters still need to be investigated, only a few work has focused on the physical origin of the material length scale [19-24].

The material length scale is usually determined by using the simulation results of mechanics behavior based on the SG theory

\footnotetext{
${ }^{1}$ Corresponding author.

Manuscript received August 27, 2019; final manuscript received November 8,
} 2019; published online November 25, 2019. Assoc. Editor: Alan Needleman. to fit the corresponding experimental measurements $[1-6,9]$. In fact, the material length scale is an intrinsic parameter. It is closely related to the physical mechanism and microstructure formation and evolution of materials at the micron scale. Most early attempts have been made to give an explanation through fundamental dislocation physics. Nix and Gao [9] identified $\ell$ as $L^{2} / b$, where $L$ is the average spacing between dislocation and $b$ is the magnitude of the Burgers vector. Al-Rub and Voyiadjis [19] pointed out that the material length scale in metals can be considered by itself as an internal variable representing the dislocation cell structure and grain size. Fleck and Shu [20] defined $\ell$ as the fiber thickness when studying the fiber-reinforced composites. Zhuk et al. [21] found the ratio between $\ell$ and the size of small-scale yielding plastic zone was 0.3 for multilayer film/matrix structures. Liu and Dunstan [22] established a connection between $\ell$ and the fundamental physical quantities via critical thickness theory. Until now, the clear physical interpretation of the material length scale still needs to be investigated.

The physical meaning of the material length scale parameters is probably that it describes a representative size of strong strain gradient effect zone near interface, or surface, or singular points (such as crack tip, etc.), when material is loaded, it should be the material parameter. We have noted that at nano- or micro-scale, one can use strain gradient theory or use the Cauchy-Born rule (CBR) method to describe the material mechanical behavior independently; therefore, we have presented the equivalent method to determine the elastic length scale parameters appeared in the elastic strain gradient theory indirectly connected with the interatomic potential parameters (or called the microscopic physical parameters).

In our previous work [25], we paid great attention to the surface energy density computations, meanwhile the elastic material length scales for typical face-centered cubic (FCC) metals were determined through an equivalent condition of the strain energy density calculated by adopting both the elastic SG theory and the CBR method based on the Lennard-Jones potential, respectively, for a physical model, the spherical expanding at nanoscale. 
In the present research, we conduct a more in-depth systematic study based on the previous work. In addition to the case of the FCC metals, the parameters $\ell$ for body-centered cubic (BCC) and hexagonal close-packed (HCP) typical metals will also be determined by linking the elastic SG theory and the CBR method. Both the standard Cauchy-Born rule (SCBR) and the higher-order Cauchy-Born rule (HCBR) will be considered and compared. The CBR is broadly used as a bridge to relate the continuum theories with typical atomic or molecular theories and expresses material energy and stress by the interatomic potential, which contains the microscopic physical parameters of the materials such as potential parameters, lattice constants, etc. Therefore, through the present method, the microscopic physical characterization for the elastic material length scale of the elastic SG theory can also be characterized and obtained.

The SG theory sometimes was called the cross-scale mechanics theory by which the mechanics behavior of ASMs which are designed starting from microstructures is characterized. The macroscopic mechanics properties of the ASMs are related to their microstructure features. One can simplify the ASMs into the equivalent homogeneous materials, and their mechanics properties need to be described by strain gradient theory in elasticity or in elastoplasticity in order to interpret size effect. As conventional material parameters, the material length parameter appeared in the strain gradient theory should be determined through experiment measurements, however as well known that this kind of experiments is very difficult to be done so that the progress of the related researches is very slowly until now. In the present research, we presented a very simple method (equivalent method) to determine the elastic length parameter appeared in the elastic strain gradient theory. We shall adopt the theories that were constructed following from the thermodynamics way under which it does not matter that the materials studied are metals or not, although we focus attention to typical metal materials in the present researches.

The paper is organized as follows. In Sec. 2, the typical theoretical models of the elastic SG theory and the CBR method are briefly reviewed. In Sec. 3, the parameters $\ell$ are characterized by adopting the spherical expanding model. The equivalent condition of linking the elastic SG theory and the CBR method is proposed. In Sec. 4, the results are analyzed and discussed for typical metal systems, the FCC, BCC, and HCP metals. The differences between the results using SCBR and HCBR, the Aifantis SG theory and the simplified Wei-Hutchinson SG theory are also compared. In Sec. 5, conclusions are given.

\section{Related Cross-Scale Theories and Methods}

2.1 Elastic Strain Gradient Theory. There are several versions of elastic SG theories, the material length scales contained therein are different from each other. There are 18 independent components ( 8 antisymmetric components and 10 symmetric components) contained in the elastic SG theory established by Mindlin [26]. For the isotropic materials, the second-order displacement gradient term corresponds to 5 non-classical material length parameters. In view of the difficulty in determining the length scale parameters, Altan and Aifantis [10] proposed a simplified SG elasticity theory based on Mindlin SG theory [26], in which only one material length scale is introduced. Gao and Park et al. [17] gave the variation equations of the theory based on the principle of energy minimization. Wei and Hutchinson [13] and Wei [14] further refined the structure of the SG theory based on the framework of the Fleck-Hutchinson SG theory [12]. In the present work, we will adopt the Aifantis SG elastic theory and the simplified Wei-Hutchinson SG elastic theory, respectively.

2.1.1 Aifantis Strain Gradient Elastic Theory. For isotropic elastic materials, the variables included in the Aifantis SG elastic theory $[10,17]$ are strain $\boldsymbol{\varepsilon}$, Cauchy stress $\boldsymbol{\sigma}$, strain gradient $\boldsymbol{\kappa}$, and couple stress $\boldsymbol{\mu}$. The tensor representation of its basic equations can be expressed as

$$
\begin{gathered}
\boldsymbol{\varepsilon}=\frac{1}{2}\left[\nabla \boldsymbol{u}+(\nabla \boldsymbol{u})^{\mathrm{T}}\right], \quad \boldsymbol{\kappa}=\frac{1}{2} \nabla\left[\nabla \boldsymbol{u}+(\nabla \boldsymbol{u})^{\mathrm{T}}\right] \\
\boldsymbol{\sigma}=\lambda(\nabla \cdot \boldsymbol{u}) \boldsymbol{I}+\mu\left[\nabla \boldsymbol{u}+(\nabla \boldsymbol{u})^{\mathrm{T}}\right], \quad \boldsymbol{\mu}=l^{2} \nabla \boldsymbol{\sigma}
\end{gathered}
$$

and the governing equation reads

$$
\left(1-l^{2} \nabla^{2}\right)[(\lambda+2 \mu) \nabla(\nabla \cdot \boldsymbol{u})-\mu \operatorname{curl}(\operatorname{curl} \boldsymbol{u})]+\boldsymbol{f}=0
$$

where $\lambda$ and $\mu$ are the Lamé constants, $\nabla, \nabla \cdot$, and $\nabla^{2}$ are gradient, divergence and Laplace operator, respectively, $\boldsymbol{u}$ is displacement tensor, and $l$ is the material length scale.

The strain energy density based on the Aifantis SG elasticity theory can be expressed as

$$
w_{A-S G}=\frac{1}{2}(\boldsymbol{\sigma}: \boldsymbol{\varepsilon}+\boldsymbol{\mu}: \boldsymbol{\kappa})
$$

2.1.2 Simplified Wei-Hutchinson Strain Gradient Elastic Theory. In the framework of the simplified Wei-Hutchinson SG elastic theory $[13,14]$, the generalized strain tensor includes the following two parts:

$$
\varepsilon_{i j}=\frac{1}{2}\left(u_{i, j}+u_{j, i}\right), \quad \eta_{i j k}=u_{k, i j}
$$

Introducing orthogonal decomposition of the strain gradient tensor,

$$
\begin{array}{cc}
\eta_{i j k}=\sum_{I=1}^{4} \eta_{i j k}^{(I)} & \left(I \neq J, \eta_{i j k}^{(I)} \eta_{i j k}^{(J)}=0\right) \\
\eta_{i j k}^{(I)}=T_{i j k l m n}^{(I)} \eta_{l m n} & I=1,4
\end{array}
$$

where $T_{i j k l m n}^{(I)}(I=1,4)$ are the projection tensors for strain gradient.

For the elastic case, the constitutive equations are [14]

$$
\begin{aligned}
\sigma_{i j} & =\frac{E}{1+\nu} \varepsilon_{i j}+\frac{E \nu}{(1-2 \nu)(1+\nu)} \varepsilon_{k k} \delta_{i j} \\
\tau_{i j k} & =2 E\left\{\sum_{I=1}^{4} l_{I}^{2} T_{i j k l m n}^{(I)}\right\} \eta_{l m n}, \quad I=1,4
\end{aligned}
$$

where $E$ and $\nu$ are the Young's modulus and Poisson's ratio, $l_{I}(I=$ $1,4)$ are the material length scales; here, we take the same value $l_{I}=l(I=1,4)$.

The strain energy density based on the simplified WeiHutchinson SG elastic theory can be expressed as

$$
w_{W-S G}=\frac{1}{2}(\boldsymbol{\sigma}: \boldsymbol{\varepsilon}+\boldsymbol{\tau}: \boldsymbol{\eta})
$$

2.2 Cauchy-Born Rule Method. In the present paper, the SCBR and the HCBR will be adopted and compared, respectively. The SCBR failed when the unconstrained atomistic deformation became inhomogeneous due to instabilities [27]. Sunyk and Steinmann [28] considered the SCBR in the classical form as the first term of a Taylor's series expansion of the deformation field and enhanced this expansion by the second quadratic term including the higher-order deformation gradient, and proposed the HCBR.

In the framework of continuum mechanics, the non-linear deformation map $\varphi(X)$ relates a point $X$ in the initial configuration before deformation and a point $x$ in the current configuration after deformation: $x=\varphi(X)$, the local deformation gradient $F$ can be expressed as

$$
F=\nabla_{X} \varphi=\frac{\partial \varphi}{\partial X}
$$

Considering a crystallite body consisting of $N$ interacting atoms, the kinematics are then typically represented by the distance vectors between two atoms labeled $i$ and $j$, i.e., $\boldsymbol{R}_{i j}$ and $\boldsymbol{r}_{i j}$ in the initial and in 
the current configuration, respectively.

$$
\boldsymbol{R}_{i j}=\boldsymbol{R}_{i}-\boldsymbol{R}_{j}, \quad \boldsymbol{r}_{i j}=\boldsymbol{r}_{i}-\boldsymbol{r}_{j}
$$

The position vectors $\boldsymbol{R}_{i}$ and $\boldsymbol{r}_{i}$ in both configurations are connected by the non-linear discrete map $\varphi\left(\boldsymbol{R}_{i}\right)$. The SCBR assumes that this discrete mapping is replaced by a continuum mapping, and the relative position of the atoms after deformation is specified by

$$
\boldsymbol{r}_{i j}=\boldsymbol{F} \cdot \boldsymbol{R}_{i j}
$$

The HCBR introduces the second-order deformation gradient $\boldsymbol{G}$ based on SCBR, and the fundamental relation can be read as

$$
\begin{aligned}
\boldsymbol{r}_{i j} & =\boldsymbol{F} \cdot \boldsymbol{R}_{i j}+\frac{1}{2} \boldsymbol{G}:\left[\boldsymbol{R}_{i j} \otimes \boldsymbol{R}_{i j}\right] \\
\boldsymbol{G} & =\nabla \boldsymbol{F}=\nabla_{\boldsymbol{X}} \nabla_{\boldsymbol{X}} \varphi
\end{aligned}
$$

The core of the CBR method used in this paper is to determine the total energy or energy density of the system. The energy of the system is a function of degrees of freedom (i.e., the position of the atoms), and the interaction between atoms is governed through potential, such as the Lennard-Jones (L-J) pair potential,

$$
\phi\left(r_{i j}\right)=4 e\left[\left(\frac{r_{0}}{r_{i j}}\right)^{12}-\left(\frac{r_{0}}{r_{i j}}\right)^{6}\right]
$$

in which two atoms $i$ and $j$ are at the relative distance $\boldsymbol{r}_{i j}$. The parameters $e$ and $r_{0}$ represent the energy at the minimum in $\phi\left(r_{i j}\right)$ and the distance to zero. The energy contribution $E_{i}$ of atom $i$ to the total energy can be obtained as

$$
E_{i}=\frac{1}{2} \sum_{j \neq i} \phi\left(r_{i j}\right)=\frac{1}{2} \sum_{j \neq i} \phi\left(\left|\boldsymbol{r}_{i j}\right|\right)
$$

The strain energy density can be expressed as

$$
w_{C B R}=\frac{\Delta E_{i}}{V_{i}}=\frac{1}{2 V_{i}} \sum_{j \neq i}\left[\phi\left(\left|\boldsymbol{r}_{i j}\right|\right)-\phi\left(\left|\boldsymbol{R}_{i j}\right|\right)\right]
$$

where $V_{i}$ denotes the effective volume around the atom $i$,

$$
V_{i}=\frac{V}{N}
$$

where $V$ is the total volume of the body and $N$ is the total number of the atoms in the body.

\section{Physical Model Selection and Solution}

In our previous work [25], we used a spherical expanding cell model and obtained the material length scales for several typical FCC metals by the linkage of results of the strain energy density based on the simplified Wei-Hutchinson SG elastic theory and the HCBR method, respectively. In the present paper, we conducted further systematic and in-depth researches on this model for the Aifantis SG elastic theory, the simplified WeiHutchinson SG elastic theory, the SCBR method as well as the HCBR method, and for typical FCC, BCC, and HCP metals, respectively.

3.1 Solution Based on Elastic Strain Gradient Theory. Considering a sphere having the radius $R$ and expanding outward by the deformation gradient $\boldsymbol{F}$ under the spherical coordinate system $(r, \theta, \varphi)$, the displacement field has the form

$$
\boldsymbol{u}=u(r) \boldsymbol{e}_{r}
$$

According to Eqs. (1)-(4), the strain energy density $w_{A-S G}$ based on the Aifantis SG elastic theory can be given by

$$
\begin{aligned}
w_{A-S G}= & \frac{1}{2}(\boldsymbol{\sigma}: \boldsymbol{\varepsilon}+\boldsymbol{\mu}: \boldsymbol{\kappa}) \\
= & \frac{1}{2}\left\{(\lambda+2 \mu) u^{\prime 2}+4 \lambda\left(\frac{u \cdot u^{\prime}}{r}\right)+4(\lambda+\mu)\left(\frac{u}{r}\right)^{2}+l_{A}^{2}\left[(\lambda+2 \mu) u^{\prime \prime 2}\right.\right. \\
& \left.\left.+4 \lambda u^{\prime \prime}\left(\frac{u^{\prime}}{r}-\frac{u}{r^{2}}\right)+4(\lambda+3 \mu)\left(\frac{u^{\prime}}{r}-\frac{u}{r^{2}}\right)^{2}\right]\right\}
\end{aligned}
$$

where $l_{A}$ is the material length scale in the Aifantis SG elastic theory.

Combining Eqs. (5)-(8), the strain energy density $w_{W-S G}$ based on the simplified Wei-Hutchinson SG elastic theory can be given by

$$
\begin{aligned}
w_{W-S G}= & \frac{1}{2}(\boldsymbol{\sigma}: \boldsymbol{\varepsilon}+\boldsymbol{\tau}: \boldsymbol{\eta}) \\
= & \frac{1}{2}\left\{(\lambda+2 \mu) u^{\prime 2}+4 \lambda\left(\frac{u \cdot u^{\prime}}{r}\right)+4(\lambda+\mu)\left(\frac{u}{r}\right)^{2}\right. \\
& \left.+2 E l_{W}^{2}\left[u^{\prime \prime 2}+6\left(\frac{u^{\prime}}{r}-\frac{u}{r^{2}}\right)^{2}\right]\right\}
\end{aligned}
$$

where $l_{W}$ is the material length scale in the simplified WeiHutchinson SG elastic theory.

The detailed derivation process can be seen in Refs. [17,25], and the general solution form of the displacement can be expressed as

$$
u_{S G}(r)=A r+\frac{B}{r^{2}}\left[(r-L) e^{r / L}+(r+L) e^{-r / L}\right]
$$

where $L=l_{A}$ for the Aifantis SG elastic theoryand $L=$ $l_{W} \sqrt{2 E /(\lambda+2 \mu)}$ for the simplified Wei-Hutchinson SG elastic theory. The undetermined constants $A$ and $B$ are different for the two SG elastic theories and can be determined by the outer boundary conditions and the equivalent conditions, which will be given in detail in Sec. 3.2.

3.2 Solution Based on Cauchy-Born Rule Method. Take the atom at the center of the sphere as the representative atom $i$, the radius at any other atom $j$ is $r$, under the spherical coordinate system, the displacement is $u(r)$. The atomic spacing vector before deformation can be written as $\boldsymbol{R}_{i j}=r \boldsymbol{e}_{r}$, and after deformation, it becomes $\boldsymbol{r}_{i j}=(r+u) \boldsymbol{e}_{r}$; therefore, the deformation gradient tensor is

$$
\boldsymbol{F}=\nabla \boldsymbol{r}_{i j}=\left(1+u^{\prime}\right) \boldsymbol{e}_{r} \boldsymbol{e}_{r}+\left(1+\frac{u}{r}\right)\left(\boldsymbol{e}_{\theta} \boldsymbol{e}_{\theta}+\boldsymbol{e}_{\varphi} \boldsymbol{e}_{\varphi}\right)
$$

The second-order deformation gradient is

$$
\begin{aligned}
\boldsymbol{G}= & \nabla \boldsymbol{F}=\nabla \nabla \boldsymbol{r}_{i j} \\
= & u^{\prime \prime} \boldsymbol{e}_{r} \boldsymbol{e}_{r} \boldsymbol{e}_{r}+\left(\frac{u^{\prime}}{r}-\frac{u}{r^{2}}\right)\left(\boldsymbol{e}_{\theta} \boldsymbol{e}_{\theta} \boldsymbol{e}_{r}+\boldsymbol{e}_{\varphi} \boldsymbol{e}_{\varphi} \boldsymbol{e}_{r}\right) \\
& +\left(1+u^{\prime}\right) \nabla\left(\boldsymbol{e}_{r} \boldsymbol{e}_{r}\right)+\left(1+\frac{u}{r}\right) \nabla\left(\boldsymbol{e}_{\theta} \boldsymbol{e}_{\theta}+\boldsymbol{e}_{\varphi} \boldsymbol{e}_{\varphi}\right)
\end{aligned}
$$

We introduce an effective deformation gradient $\tilde{\boldsymbol{F}}$, the SCBR and $\mathrm{HCBR}$ can be written in the unified form

$$
\boldsymbol{r}_{i j}=\tilde{\boldsymbol{F}} \cdot \boldsymbol{R}_{i j}
$$

For the spherical expanding model, the displacement based on the CBR can be expressed as

$$
u_{C B R}(r)=\tilde{F}_{r r} \cdot r-r
$$

where $\tilde{F}_{r r}$ is the radial component of $\tilde{\boldsymbol{F}}$. 
For SCBR, we have $\tilde{\boldsymbol{F}}=\boldsymbol{F}$; therefore,

$$
\tilde{F}_{r r}=1+u_{C B R}^{\prime}(r)
$$

Substituting Eq. (24) into the above equation, we can get $\tilde{F}_{r r}^{\prime}=0$. That is to say, the radial component of $\tilde{\boldsymbol{F}}$ is a constant, combining with the Eq. (21), we obtain

$$
\tilde{\boldsymbol{F}}=\left[\begin{array}{lll}
f & 0 & 0 \\
0 & f & 0 \\
0 & 0 & f
\end{array}\right]
$$

where $f$ is the spherical expanding ratio, a constant.

For HCBR, the effective deformation gradient can expressed as

$$
\begin{aligned}
\tilde{\boldsymbol{F}} & =\boldsymbol{F}+\frac{1}{2} \boldsymbol{G} \cdot \boldsymbol{R}_{i j} \\
& =\left(1+u^{\prime}+\frac{1}{2} u^{\prime \prime} r\right) \boldsymbol{e}_{r} \boldsymbol{e}_{r}+\left(1+\frac{u+u^{\prime} r}{2 r}\right)\left(\boldsymbol{e}_{\theta} \boldsymbol{e}_{\theta}+\boldsymbol{e}_{\varphi} \boldsymbol{e}_{\varphi}\right)
\end{aligned}
$$

and its the radial component is

$$
\tilde{F}_{r r}=1+u_{C B R}^{\prime}(r)+\frac{1}{2} u_{C B R}^{\prime \prime}(r) \cdot r
$$

Substituting Eq. (24) into the above equation, we can get $\tilde{F}_{r r}^{\prime \prime} \cdot r+4 \tilde{F}_{r r}^{\prime}=0$, which solution is $\tilde{F}_{r r}=\lambda_{0} \cdot r^{-3}+\lambda_{1}$, where $\lambda_{0}, \lambda_{1}$ are constants. Introducing a dimensionless constant $\lambda_{2}=\lambda_{0} / a^{3}$, where $a$ is the lattice size. The radial component of $\tilde{\boldsymbol{F}}$ becomes $\tilde{F}_{r r}=\lambda_{1}+\lambda_{2} \cdot\left(a^{3} / r^{3}\right)$. Combining with the Eq. (27), we obtain

$$
\tilde{\boldsymbol{F}}=\left[\begin{array}{ccc}
\lambda_{1}+\lambda_{2} \cdot\left(a^{3} / r^{3}\right) & 0 & 0 \\
0 & \lambda_{1}-\lambda_{2} \cdot\left(a^{3} / 2 r^{3}\right) & 0 \\
0 & 0 & \lambda_{1}-\lambda_{2} \cdot\left(a^{3} / 2 r^{3}\right)
\end{array}\right]
$$

Here, the interatomic potential adopts L-J pair potential, Eq. (13), and the strain energy density based on the CBR method, Eq. (15), can be rewritten as

$$
\begin{aligned}
w_{C B R}= & \frac{\Delta E_{i}\left(\widetilde{\boldsymbol{F}} ; \boldsymbol{R}_{i j}\right)}{V_{i}}=\frac{\frac{1}{2} \sum_{j \neq i} \Delta \phi_{i j}}{V_{i}} \\
= & \frac{N}{2 V} \sum_{j \neq i}\left\{4 e\left[\left(\frac{r_{0}}{r_{i j}}\right)^{12}-\left(\frac{r_{0}}{r_{i j}}\right)^{6}\right]-4 e\left[\left(\frac{r_{0}}{R_{i j}}\right)^{12}-\left(\frac{r_{0}}{R_{i j}}\right)^{6}\right]\right\} \\
= & \frac{N}{2 V} \sum_{j \neq i}\left\{4 e\left[\left(\frac{r_{0}}{\left|\widetilde{\boldsymbol{F}} \cdot \boldsymbol{R}_{i j}\right|}\right)^{12}-\left(\frac{r_{0}}{\left|\widetilde{\boldsymbol{F}} \cdot \boldsymbol{R}_{i j}\right|}\right)^{6}\right]\right. \\
& \left.-4 e\left[\left(\frac{r_{0}}{\left|\boldsymbol{R}_{i j}\right|}\right)^{12}-\left(\frac{r_{0}}{\left|\boldsymbol{R}_{i j}\right|}\right)^{6}\right]\right\}
\end{aligned}
$$

In the CBR method, before an atomic potential is used, the only thing needs to be done is to determine the deformation gradients. For the spherical expanding problem in the present research, it can be done by equivalent conditions, as seen Sec. 3.3. Then, we can systematically analyze the effect of the ratio of spherical radius to the lattice size, $\mathrm{R} / \mathrm{a}$, on the results.

3.3 Equivalent Method to Determine the Material Length Scales. For the spherical expanding model, the outer boundary conditions and the equivalent conditions can be used as follows:

$$
\left\{\begin{aligned}
\left.u_{S G}(r)\right|_{r=R} & =\left.u_{C B R}(r)\right|_{r=R} \\
\left.\frac{d u_{S G}(r)}{d r}\right|_{r=R} & =\left.\frac{d u_{C B R}(r)}{d r}\right|_{r=R} \\
\bar{w}_{S G} & =w_{C B R}
\end{aligned}\right.
$$

where $\bar{w}_{S G}$ are the average strain energy density of the whole sphere based on the SG theory and can be obtained by

$$
\bar{w}_{S G}=\frac{\int_{V} w_{S G}(r) d V}{V}=\frac{\int_{0}^{2 \pi} d \varphi \int_{0}^{\pi} \sin \theta d \theta \int_{0}^{R} w_{S G}(r) r^{2} d r}{V}
$$

Using Eq. (31), one can obtain two unknown constants A and B in Eq. (20) as well as materials length scale parameter.

It is worth noting that at nano-meter/micron-meter scale, the strain gradient (second-order deformation gradient) effect is considerably large. Since the SCBR method is based on the first-order deformation gradient, while the HCBR method considers the second-order deformation gradients; therefore, the HCBR method can be considered as a more accurate method than the SCBR method in determining the length scale parameters through the equivalent method. Since the elastic strain gradient theory corresponds to small deformation case, we take the same radius and same radius deformation gradient values as in the strain gradient elastic theory solution for spherical cell model in order to confirm the CBR method corresponding to the small deformation case.

\section{Results and Discussion}

In the solution process according to the equivalent method from Eq. (31), all known parameters taken here are shown in Table 1.

The dimensionless strain energy density $\left(a^{3} / e\right) w_{C B R}$ based on SCBR for $\mathrm{Cu}$ is shown in Fig. 1. We can see that, for different deformation gradient, the values of the strain energy density gradually stabilize with the sphere radius increasing, and they become independent of the spherical radius when the radius is larger than about 10 times the lattice size $a$, which is similar to the results calculated by HCBR in literature [25]. The same conclusions have been drawn from the calculation of the other typical metal material systems. From Fig. 1, when spherical radius $R$ was larger than about five times the lattice size of metal $a$, the energy density calculated from the CBR method is independent of the spherical radius $R$, and its variations with radius change ratio $f$ are from increasing at first stage to decreasing at the second stage.

Therefore, in the present research, we will choose a sufficiently large spherical radius after the strain energy density is stable. The results shown in the present paper corresponds to taking as $R / a=$ 10. We have also checked at two cases of $R / a=15$ and 20 , the results are very near the case of $R / a=10$, and the differences are within $5 \%$

Table 1 The related microscopic physics parameters and macroscopic mechanical parameters for typical FCC, BCC, and HCP metals adopted in the present research

\begin{tabular}{lccccc}
\hline \hline & $\begin{array}{c}\text { Young's } \\
\text { modulus } \\
\text { Metals }\end{array}$ & $\begin{array}{c}\text { Poisson's } \\
\text { ratio }\end{array}$ & $\begin{array}{c}\text { Lattice } \\
\text { size } a \\
(\AA)\end{array}$ & $\begin{array}{c}\text { L-J } \\
\text { potential } \\
\text { parameter } r_{0} \\
(\AA)\end{array}$ & $\begin{array}{c}\text { L-J } \\
\text { potential } \\
\text { parameter } e \\
(\mathrm{eV})\end{array}$ \\
\hline $\mathrm{Cu}$ & 119 & 0.33 & 3.61 & 2.28 & 0.42 \\
$\mathrm{Ni}$ & 200 & 0.32 & 3.52 & 2.22 & 0.53 \\
$\mathrm{Al}$ & 70 & 0.35 & 4.05 & 2.55 & 0.41 \\
$\mathrm{Ag}$ & 83 & 0.33 & 4.09 & 2.57 & 0.35 \\
$\mathrm{Pd}$ & 121 & 0.39 & 3.89 & 2.45 & 0.47 \\
$\mathrm{Fe}$ & 211 & 0.29 & 2.93 & 2.38 & 0.52 \\
$\mathrm{Cr}$ & 279 & 0.21 & 2.91 & 2.34 & 0.50 \\
$\mathrm{~W}$ & 411 & 0.28 & 3.17 & 2.56 & 1.07 \\
$\mathrm{Mo}$ & 325 & 0.29 & 3.15 & 2.55 & 0.84 \\
$\mathrm{~V}$ & 128 & 0.37 & 3.03 & 2.46 & 0.65 \\
$\mathrm{Mg}$ & 45 & 0.29 & 3.21 & 2.94 & 0.18 \\
$\mathrm{Zn}$ & 105 & 0.25 & 2.67 & 2.45 & 0.16 \\
$\mathrm{Cd}$ & 50 & 0.30 & 2.98 & 2.73 & 0.14 \\
$\mathrm{Co}$ & 211 & 0.31 & 2.51 & 2.30 & 0.51 \\
$\mathrm{Be}$ & 318 & 0.02 & 2.29 & 2.09 & 0.39 \\
\hline \hline
\end{tabular}




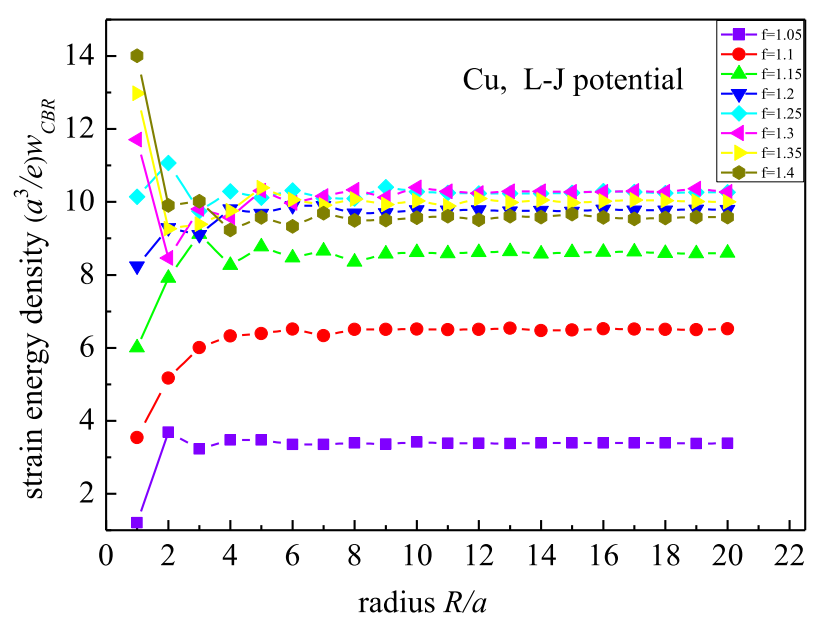

Fig. 1 Relations of the average strain energy density with a spherical radius

From Fig. 1, when the ratio of spherical radius to the lattice size, $R / a$, is smaller than about 2 , the energy density calculated is waved, this is because that within the sphere, the atoms are very few, and the distribution regular is poor, so that the law of the deformation gradient is also poor.

For the combination of both the SCBR method and the Aifantis/ simplified Wei-Hutchinson SG elastic theories, solving the above Eqs. (17)-(32), one can obtain the corresponding unknown constants $A$ and $B$ in Eq. (20) and the material length scales $l_{A}$ and $l_{W}$. The values of $l_{A}$ for FCC, BCC, and HCP metals are given in Fig. 2. In order to conform the small deformation, within the typical small deformation region $(1.01,1.08)$ of the radius deformation gradient $f$, length scale values vary with $f$. It can be seen that the values of the material length scale vary with the deformation gradient. The values of $l_{A}$ for typical FCC metals $(\mathrm{Cu}, \mathrm{Al}, \mathrm{Ag}, \mathrm{Ni}, \mathrm{Pd})$ are in the scope of $(0.5,3) \mu \mathrm{m}$, for typical BCC metals (Fe, Cr, W, Mo, V) are in the scope of $(0.2,1.5) \mu \mathrm{m}$, and for typical HCP metals $(\mathrm{Mg}$, $\mathrm{Zn}, \mathrm{Cd}, \mathrm{Co}, \mathrm{Be})$ are in the scope of $(0.1,1) \mu \mathrm{m}$. Within the spherical expanding deformation gradient $(1.01,1.09)$, the length scale parameters for the above typical metal systems are all on the order of micrometers. We also find that the range of the material length scales of these materials has the following relationship: $\mathrm{FCC}>\mathrm{BCC}>\mathrm{HCP}$.

The average values of the material length scales $\bar{l}_{A}$ and $\bar{l}_{W}$ calculated through the equivalent connection between SCBR and Aifantis/simplified Wei-Hutchinson SG theory are listed in Table 2. We can see that the length scales in the two theories for the same metal are similar in average values, and there is a common law, that is in FCC metals, $\mathrm{Al}$ has the largest value and $\mathrm{Pd}$ has the smallest; in BCC metals, $\mathrm{Cr}$ and $\mathrm{V}$ have the largest values and Mo has the smallest; in HCP metals, $\mathrm{Cd}$ is the largest, and $\mathrm{Mg}$ is the smallest.

For the combination of both the HCBR method and the Aifantis/ Wei-Hutchinson SG elastic theories, solving the above Eqs. (17)(32), one can obtain the corresponding unknown constants $A$ and $B$ in Eq. (20) and the material length scales $l_{A}$ and $l_{W}$, as shown in Fig. 3. The average values are also listed in Table 3 . The range of the values for FCC metals is in $1.2 \mu \mathrm{m} \sim 4.8 \mu \mathrm{m}$, BCC metals is in $0.2 \mu \mathrm{m} \sim 2.5 \mu \mathrm{m}$, HCP metals is in $0.1 \mu \mathrm{m} \sim 2 \mu \mathrm{m}$, and the common law in the above paragraph is also obtained.

Compared with the results of the SCBR, the values calculated by the HCBR are generally larger. For different metals in the typical metal systems, the order of the values calculated by SCBR and $\mathrm{HCBR}$ is consistent, such as $\mathrm{Al}$ has the largest material length scale in FCC metals, etc., and the relationship of FCC $>$ BCC $>$ HCP is satisfied. For the same metal, the material length scales in Aifantis SG elastic theory and Wei-Hutchinson SG elastic theory are almost the same. We can see that the material length scales can be characterized by the microscopic physical parameters of
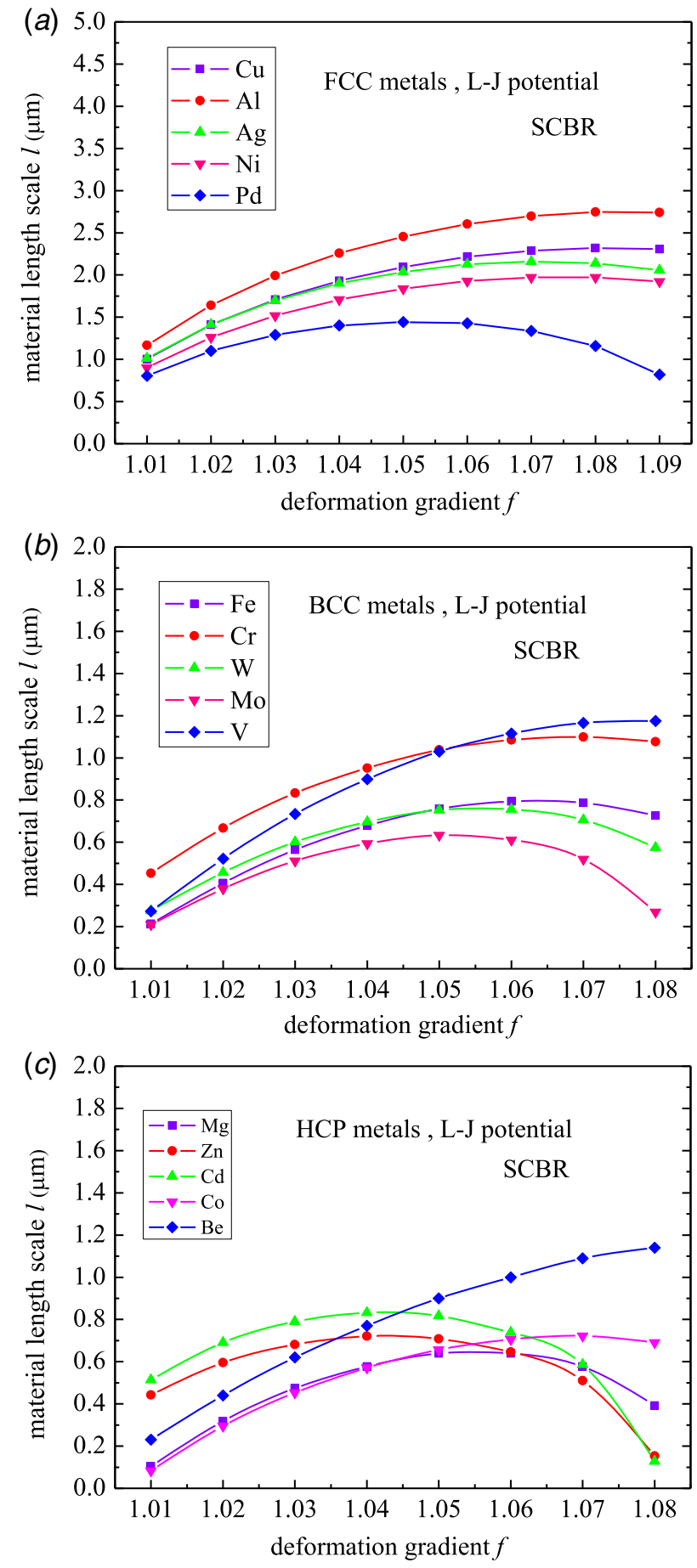

Fig. 2 Material length scales for typical metals calculated through the equivalent connection between SCBR and Aifantis SG elastic theory: (a) FCC, (b) BCC, and (c) HCP

Table 2 The average values of the material length scales for several typical metals obtained by SCBR

\begin{tabular}{lcccccccc}
\hline \hline FCC & $\bar{l}_{A}(\mu \mathrm{m})$ & $\bar{l}_{W}(\mu \mathrm{m})$ & BCC & $\bar{l}_{A}(\mu \mathrm{m})$ & $\bar{l}_{W}(\mu \mathrm{m})$ & $\mathrm{HCP}$ & $\bar{l}_{A}(\mu \mathrm{m})$ & $\bar{l}_{W}(\mu \mathrm{m})$ \\
\hline $\mathrm{Cu}$ & 1.92 & 1.44 & $\mathrm{Fe}$ & 0.62 & 1.08 & $\mathrm{Mg}$ & 0.46 & 0.42 \\
$\mathrm{Ni}$ & 1.67 & 1.27 & $\mathrm{Cr}$ & 0.90 & 1.20 & $\mathrm{Zn}$ & 0.56 & 0.75 \\
$\mathrm{Al}$ & 2.26 & 1.55 & $\mathrm{~W}$ & 0.60 & 0.91 & $\mathrm{Cd}$ & 0.64 & 0.89 \\
$\mathrm{Ag}$ & 1.84 & 1.21 & $\mathrm{Mo}$ & 0.47 & 0.76 & $\mathrm{Co}$ & 0.52 & 0.47 \\
$\mathrm{Pd}$ & 1.20 & 1.03 & $\mathrm{~V}$ & 0.86 & 1.51 & $\mathrm{Be}$ & 0.77 & 0.43 \\
\hline \hline
\end{tabular}



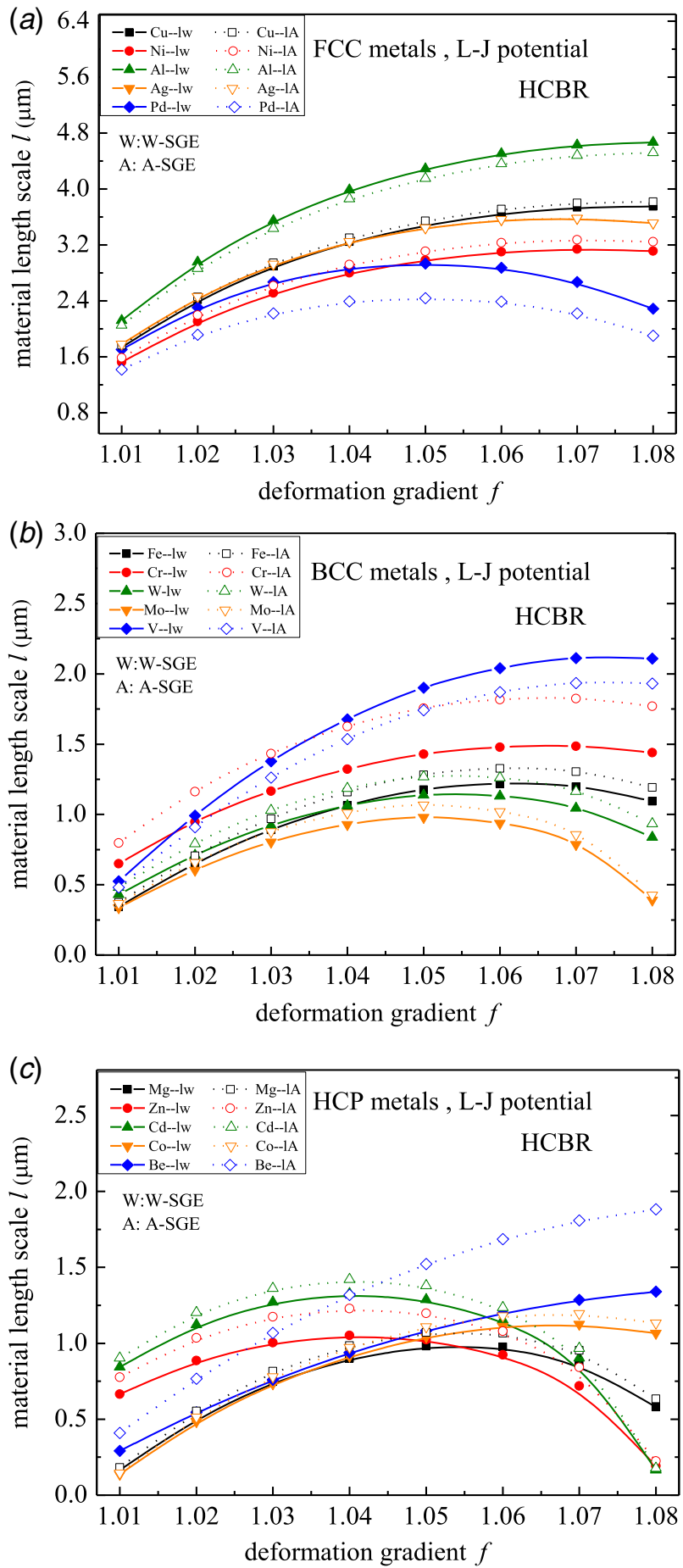

Fig. 3 Material length scales for typical metals calculated through the equivalent connection between HCBR and Aifantis/ Wei-Hutchinson SG elastic theory: (a) FCC, (b) BCC, and (c) HCP

Table 3 The average values of the material length scales for several typical metals obtained by HCBR

\begin{tabular}{lcccccccc}
\hline \hline FCC & $\bar{l}_{A}(\mu \mathrm{m})$ & $\bar{l}_{W}(\mu \mathrm{m})$ & BCC & $\bar{l}_{A}(\mu \mathrm{m})$ & $\bar{l}_{W}(\mu \mathrm{m})$ & HCP & $\bar{l}_{A}(\mu \mathrm{m})$ & $\bar{l}_{W}(\mu \mathrm{m})$ \\
\hline $\mathrm{Cu}$ & 3.17 & 3.12 & Fe & 1.04 & 0.96 & $\mathrm{Mg}$ & 0.78 & 0.72 \\
$\mathrm{Ni}$ & 2.77 & 2.65 & $\mathrm{Cr}$ & 1.52 & 1.24 & $\mathrm{Zn}$ & 0.94 & 0.81 \\
$\mathrm{Al}$ & 3.72 & 3.82 & $\mathrm{~W}$ & 1.01 & 0.91 & $\mathrm{Cd}$ & 1.22 & 1.01 \\
$\mathrm{Ag}$ & 3.05 & 3.05 & $\mathrm{Mo}$ & 0.78 & 0.72 & $\mathrm{Co}$ & 0.88 & 0.82 \\
$\mathrm{Pd}$ & 2.11 & 2.53 & $\mathrm{~V}$ & 1.45 & 1.58 & $\mathrm{Be}$ & 1.20 & 0.93 \\
\hline \hline
\end{tabular}

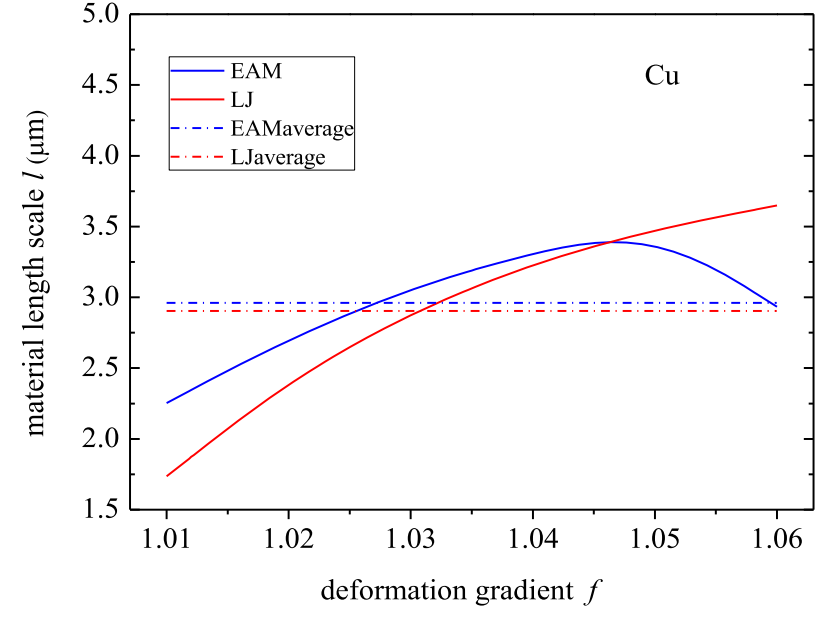

Fig. 4 A comparison of $\mathrm{Cu}$ length scale parameters predicted using the equivalent method based on both $L-J$ potential and EAM potential

the materials. For the relationship among length scale parameters $\mathrm{FCC}>\mathrm{BCC}>\mathrm{HCP}$, the main reason is probably that the three kinds of the crystals display the different magnitudes of volume energies under loading based on the L-J potential. Certainly, different mechanical parameters such as Young's modulus and Poisson ratios of the three kinds of crystals cause the different deformation energies based on the elastic strain gradient theory is another reason.

As a comparison with the $\mathrm{L}-\mathrm{J}$ potential case, we consider the embedded atom method (EAM) potential for copper here. EAM potential is more suitable for metal materials, which was proposed by Mishin et al. [29] to describe copper, and was improved by Hijazi and Park [30] for FCC metals. For the present sphere model, the material length scale parameters of $\mathrm{Cu}$ are calculated using the HCBR method based on EAM potential with Hijazi and Park' version by the equivalent method. The comparison of results based on both L-J potential and EAM potential is shown in Fig. 4. The average values of the length scale parameters within the effective region $1.01<F<1.06$ of the deformation gradient based on both $\mathrm{L}-\mathrm{J}$ and EAM potentials are very near each other, $2.89 \mu \mathrm{m}$ and $2.92 \mu \mathrm{m}$.

From above analysis, it is worth noting that in order to determine the length scale parameters in elastic strain gradient theory, we presented the equivalent method simply connecting two fully different theories through energy density equivalency, one is the continuum cross-scale mechanics method based on the elastic strain gradient concept, other one is the CBR method based on the interatomic potential concept. We have an expectation to obtain the average values of the elastic material length scale parameters within an effective deformation gradient region. From this kind of equivalent method, although the material lengths scale change with deformation gradient, we are more concerned with their average values within the effective deformation gradient region. From the above analysis, we found that the strain gradient elasticity effect is important, which is consistent with that shown in Ref. [1].

It is also worth noting that in the present paper, we considered a kind of perfect case (defect-free) in order to determine the material parameters (length scales in elastic strain gradient theory). After the material parameters are determined, we can further use the theory to analyze a true engineering problem, including defect, crack, etc.

\section{Conclusion}

In the present research, the values of the material length scales are obtained for several typical metal systems, such as FCC, BCC, and HCP metals, through using the energy density equivalency based on the elastic strain gradient theory and the Cauchy-Born rule method. The ranges of the material length scale obtained by the present 
microscopic physics method are at the micron level. Through observing and comparing the results based on the SCBR method and on the HCBR method, we think that the HCBR method is more accurate because it can describe both first-order and second-order effects of deformation gradients effectively, especially when the non-uniform gradient becomes large. For the typical metals, there exists a relation on the values of the material length scale parameters that $\mathrm{FCC}>\mathrm{BCC}>\mathrm{HCP}$. For the same metal, the values of the length scale parameters included in different elastic strain gradient theories obtained from the method are similar. It shows that the material length scale parameters are closely related to the intrinsic microscopic physical properties of the material itself, which is significant to probably make clear the physical meanings of the material length scales and promote the development and application of the cross-scale mechanics theory.

\section{Acknowledgment}

This work is supported by the National Natural Science Foundation of China (Nos. 11972347, 11502273, 11432014, 11672301, 11890681, and 11521202) and the Strategic Priority Research Program of the Chinese Academy of Sciences (Grant No. XDB22040501).

\section{References}

[1] Lam, D. C. C., Yang, F., Chong, A. C. M., Wang, J., and Dong, P., 2003, "Experiments and Theory in Strain Gradient Elasticity," J. Mech. Phys. Solids, 51(8), pp. 1477-1508.

[2] Stolken, J. S., and Evans, A. G., 1998, "A Microbend Test Method for Measuring the Plasticity Length Scale," Acta Mater., 46(14), pp. 5109-5115.

[3] Haque, M. A., and Saif, M. T. A., 2003, "Strain Gradient Effect in Nanoscale Thin Films," Acta Mater., 51(11), pp. 3053-3061.

[4] Hayashi, I., Sato, M., and Kuroda, M., 2011, "Strain Hardening in Bent Copper Foils,” J. Mech. Phys. Solids, 59(9), pp. 1731-1751.

[5] Liu, D., He, Y., Dunstan, D. J., Zhang, B., Gan, Z., Hu, P., and Ding, H., 2013, "Toward a Further Understanding of Size Effects in the Torsion of Thin Metal Wires: an Experimental and Theoretical Assessment," Int. J. Plast., 41, pp. 30-52.

[6] Fleck, N. A., Muller, G. M., Ashby, M. F., and Hutchinson, J. W., 1994, "Strain Gradient Plasticity Theory and Experiment," Acta Metall. Mater., 42(2), pp. 475-487.

[7] Ma, Z. S., Zhou, Y. C., Long, S. G., and Lu, C., 2012, "On the Intrinsic Hardness of a Metallic Film/Substrate System: Indentation Size and Substrate Effects," Int. J. Plast., 34, pp. 1-11.

[8] Zhu, T. T., Bushby, A. J., and Dunstan, D. J., 2008, "Size Effect in the Initiation of Plasticity for Ceramics in Nanoindentation,” J. Mech. Phys. Solids, 56(4), pp. 1170-1185.
[9] Nix, W. D., and Gao, H. J., 1998, "Indentation Size Effects in Crystalline Materials: A Law for Strain Gradient Plasticity," J. Mech. Phys. Solids, 46(3), pp. $411-425$.

[10] Altan, B. S., and Aifantis, E. C., 1997, "On Some Aspects in the Special Theory of Gradient Elasticity," J. Mech. Behav. Mater., 8(3), pp. 231-282.

[11] Aifantis, E. C., 1999, "Strain Gradient Interpretation of Size Effects," Int. J. Fract., 95(1/4), pp. 299-314.

[12] Fleck, N. A., and Hutchinson, J. W., 1997, "Strain Gradient Plasticity," Adv. Appl. Mech., 33, pp. 295-361.

[13] Wei, Y. G., and Hutchinson, J. W., 1997, "Steady-state Crack Growth and Work of Fracture for Solids Characterized by Strain Gradient Plasticity," J. Mech. Phys. Solids, 45(8), pp. 1253-1273.

[14] Wei, Y. G., 2006, "A new Finite Element Method for Strain Gradient Theories and Applications to Fracture Analyses," Eur. J. Mech. A/Solids, 25(6), pp. 897-913.

[15] Gao, H., Huang, Y., Nix, W. D., and Hutchinson, J. W., 1999, "Mechanism-based Strain Gradient Plasticity-I Theory," J. Mech. Phys. Solids, 47(6), pp. 1239-1263.

[16] Huang, Y., Gao, H., Nix, W. D., and Hutchinson, J. W., 2000, "MechanismBased Strain Gradient Plasticity-II Analysis," J. Mech. Phys. Solids, 48(1), pp. 99-128.

[17] Gao, X. L., Park, S. K., and Ma, H. M., 2009, "Analytical Solution for a Pressurized Thick-Walled Spherical Shell Based on a Simplified Strain Gradient Elasticity Theory," Math. Mech. Solids, 14(8), pp. 747-758.

[18] Lubarda, V. A., 2016, "On the Recoverable and Dissipative Parts of Higher Order Stresses in Strain Gradient Plasticity," Int. J. Plast., 78, pp. 26-43.

[19] Al-Rub, A., and Voyiadjis, R. K., and Z, G., 2006, "A Physically Based Gradient Plasticity Theory,” Int. J. Plast., 22(4), pp. 654-684.

[20] Fleck, N. A., and Shu, J. Y., 1995, "Microbuckle Initiation in Fibre Composites: A Finite Element Study," J. Mech. Phys. Solids, 43(12), pp. 1887-1918.

[21] Zhuk, A. V., Evans, A. G., and Hutchinson, J. W., 1998, "The Adhesion Energy Between Polymer Thin Films and Self-Assembled Monolayers," J. Mater. Res., 13(12), pp. 3555-3564.

[22] Liu, D., and Dunstan, D. J., 2017, "Material Length Scale of Strain Gradient Plasticity: A Physical Interpretation,” Int. J. Plast., 98, pp. 156-174.

[23] Dunstan, D. J., 2016, "Validation of a Phenomenological Strain-Gradient Plasticity Theory," Phil. Mag. Lett., 96(8), pp. 305-312.

[24] Zhang, X., and Aifantis, K., 2015, "Interpreting the Internal Length Scale in Strain Gradient Plasticity," Rev. Adv. Mat. Sci., 41(1-2), pp. 72-83.

[25] Song, J. R., Liu, J. Y., Ma, H. S., Liang, L. H., and Wei, Y. G., 2014, "Determinations of Both Length Scales and Surface Elastic Parameters for fcc Metals," C. R. Mecanique, 342(5), pp. 315-325.

[26] Mindlin, R. D., 1965, "Second Gradient of Strain and Surface-Tension in Linear Elasticity," Int. J. Solids Struct., 1(4), pp. 417-438.

[27] Friesecke, G., and Theil, F., 2002, "Validity and Failure of the Cauchy-Born Hypothesis in a Two-Dimensional Mass-Spring Lattice," J. Nonlinear Sci., 12(5), pp. 445-478.

[28] Sunyk, R., and Steinmann, P., 2003, "On Higher Gradients in Continuum-Atomistic Modelling," Int. J. Solids Struct., 40(24), pp. 6877-6896.

[29] Mishin, Y., Mehl, M. J., Papaconstantopoulos, D. A., Voter, A. F., and Kress, J. D., 2001, "Structural Stability and Lattice Defects in Copper: Ab Initio, Tight-Binding, and Embedded-Atom Calculations," Phys. Rev. B, 63(22), p. 224106 .

[30] Hijazi, I. A., and Park, Y. H., 2009, "Consistent Analytic Embedded Atom Potential for Face-Centered Cubic Metals and Alloys," J. Mater. Sci. Technol., 25(6), pp. 835-846. 